\title{
Lead Selenium Colloidal Quantum Dots for 400-2600nm Broadband Photodetectors
}

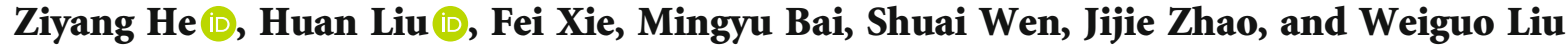 \\ School of Opto-Electronic Engineering, Xi'an Technological University, Xi'an 710021, China \\ Correspondence should be addressed to Huan Liu; liuhuan@xatu.edu.cn
}

Received 12 October 2021; Accepted 20 December 2021; Published 4 February 2022

Academic Editor: Meiyong Liao

Copyright (C) 2022 Ziyang He et al. This is an open access article distributed under the Creative Commons Attribution License, which permits unrestricted use, distribution, and reproduction in any medium, provided the original work is properly cited.

\begin{abstract}
By the photodetector manufactured using traditional semiconductor materials, such as $\mathrm{HgCdTe}$ and InGaAs, it is difficult to broaden the application range of such photodetectors due to their high cost and complex manufacturing process. PbSe colloidal quantum dots (CQDs) have the potential to shift the working range of photodetector from visible to infrared wavelength region, and it also has high photoresponsivity. Herein, we report the characterization of PbSe CQDs synthesized using a facile solution process, as well as the relationship between the size of nanocrystal and the reaction temperature. The films of PbSe CQDs are deposited using the layer-by-layer (LbL) spin-coating method, which is then used to fabricate the photoconductive device. The fabricated device is found to have an efficient response in a broad spectrum range of $400-2600 \mathrm{~nm}$. The device maintains good responsivity of $\sim 320 \mathrm{~mA} / \mathrm{W}$ at room temperature. Its external quantum efficiency was quite high in the shorter wavelength infrared region, and it has approximately $14 \%$ external quantum efficiency (EQE) at $2520 \mathrm{~nm}$. The device demonstrated excellent performance, confirming that PbSe colloidal quantum dots is a promising material for future broadband spectrum photodetectors.
\end{abstract}

\section{Introduction}

In recent years, the broadband spectrum photodetectors have piqued the interest of researchers working in a variety of fields, including environmental monitoring, biological sensing, and digital cameras [1-5]. Generally, photodetectors that can operate in a broad spectrum range, from visible to shortwavelength infrared (SWIR) region, are required for these applications. However, most photodetectors are not capable to cover such a broad wavelength range. Some commercial photodetectors that are made of silicon ( $\mathrm{Si}$ ) or indiumgallium-arsenide (InGaAs) have been used to detect visible, NIR, or SWIR photons. The lack of suitable materials that can absorb light ranging from the visible to SWIR has hampered the development of broad-spectrum photodetectors.

Traditional semiconductors, such as InGaAs and HgCdTe, are commonly used to fabricate near-to-mid infrared (IR) photodetectors. However, due to the high manufacturing costs and complex manufacturing process, it is difficult to expand the applications of such photodetectors $[6,7]$. Over the past decades, semiconductor colloidal quantum dots (CQDs) have received considerable attention owing to their unique size-dependent properties and lowcost fabrication process, and ease of their implementation in several optoelectronic devices [8-11]. One of the most significant advantages of lead selenium CQDs originates from their solution-process fabrication technique: it will bond nicely to the substrate, thus CQDs are almost unlimited in terms of substrate types, and they can be readily deposited by spin-coating, spray-casting, and inkjet printing techniques. Therefore, as a result of the simple manufacturing process, it is a cost-effective method for large-scale photodetector fabrication $[12,13]$.

However, the photodetector based on the integration of $\mathrm{Si}$ and $\mathrm{PbS}$ QDs did not demonstrate good performance. For instance, Xiao et al. fabricated a colloidal quantum dot heterojunction-based infrared photodetector. This device has an EQE of $40 \%$ when using QDs with the longest absorption wavelength of $1600 \mathrm{~nm}$ [14]. Also, a PbSe colloidal quantum dot-based infrared photodetector with room temperature responsivities of $64.17 \mathrm{~mA} / \mathrm{W}$ has been reported, but with a narrow optical spectrum ranging from 


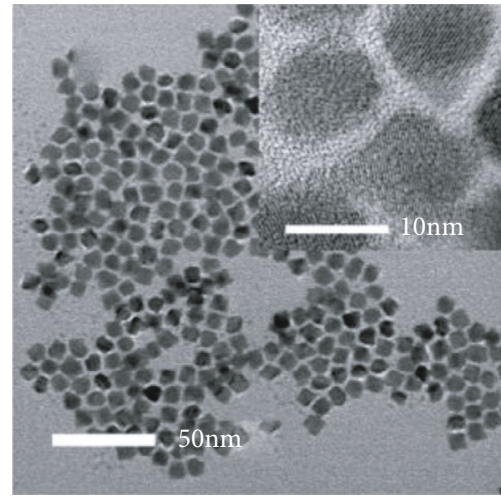

(a)

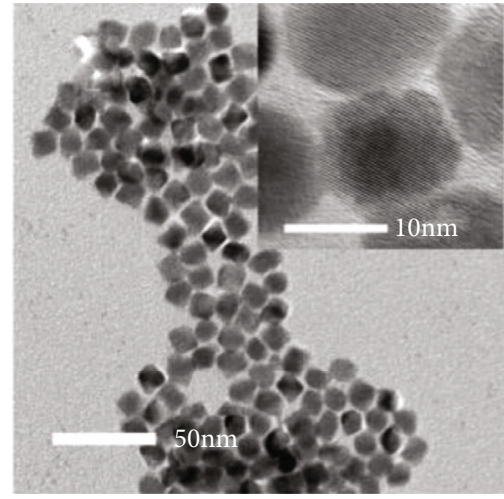

(c)

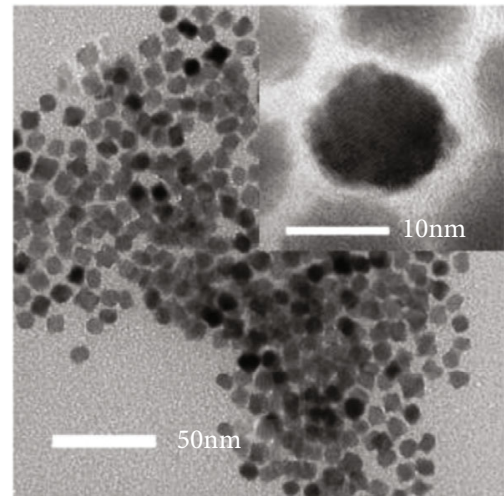

(e)

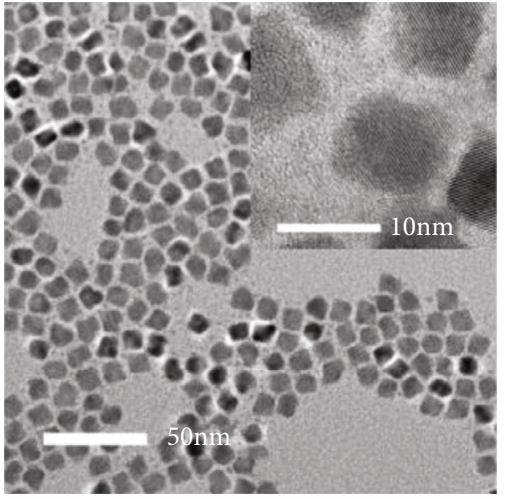

(b)

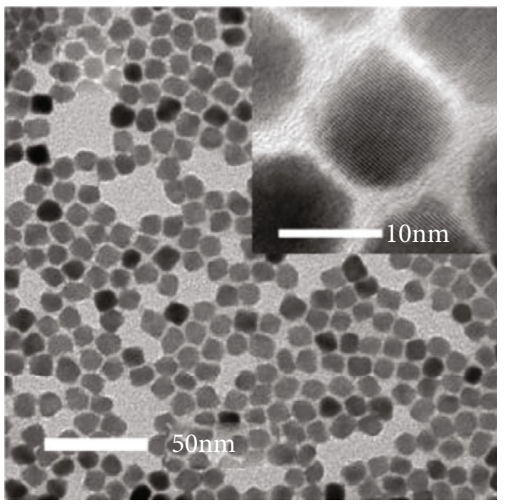

(d)

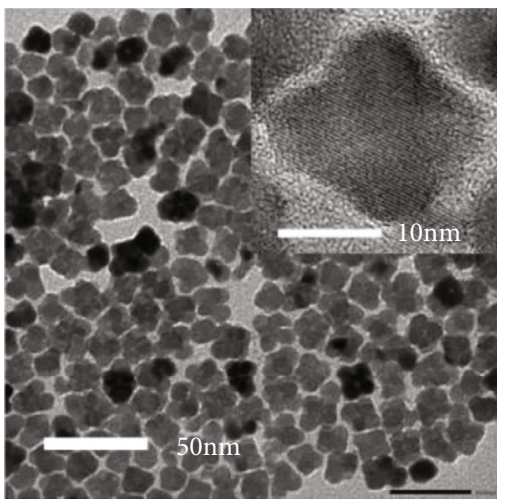

(f)

FIgure 1: The same magnification TEM images of PbSe CQDs, reaction temperature of 5 min for (a) 140, (b) 150, (c) 160, (d) 170, (e) 180, and (f) $190^{\circ} \mathrm{C}$, respectively.

400 to $1600 \mathrm{~nm}$ [15]. However, wider wavelength nearinfrared photodetectors are required for various applications, such as night glow-assisted imaging, gas alarms, and crop moisture detection.

This paper reports the synthesis and characterization of PbSe CQD developed by a facile solution process. The fabricated photoconductive device worked efficiently over the wavelength range of $400 \mathrm{~nm}$ to $2600 \mathrm{~nm}$. It is demonstrated that the synthesized PbSe CQDs can obtain optical responsivities of $320 \mathrm{~mA} / \mathrm{W}$ at $2520 \mathrm{~nm}$ at room temperature and higher than $14 \%$ external quantum efficiency in the nearinfrared region. The proposed device is expected to replace the existing near-infrared (IR) photodetector and serve as a benchmark for future broad-spectrum photodetectors.

\section{Experimental}

2.1. Materials. Lead oxide yellow (PbO, 99.999\%), selenium (Se, 99.999\%), oleic acid (OA), 1-octadecene (ODE, $90.0+\%$ ), Tri-n-octylphosphine (TOP, 90\%), ethanol (99.9\%), methanol (99.9\%), 1,2-ethanedithiol (EDT, 97\%), hexane (99.9\%), hydrochloric acid (99.9\%), and cyclohexane (99.5\%) were purchased from Aladdin. All these chemicals were used as received. 

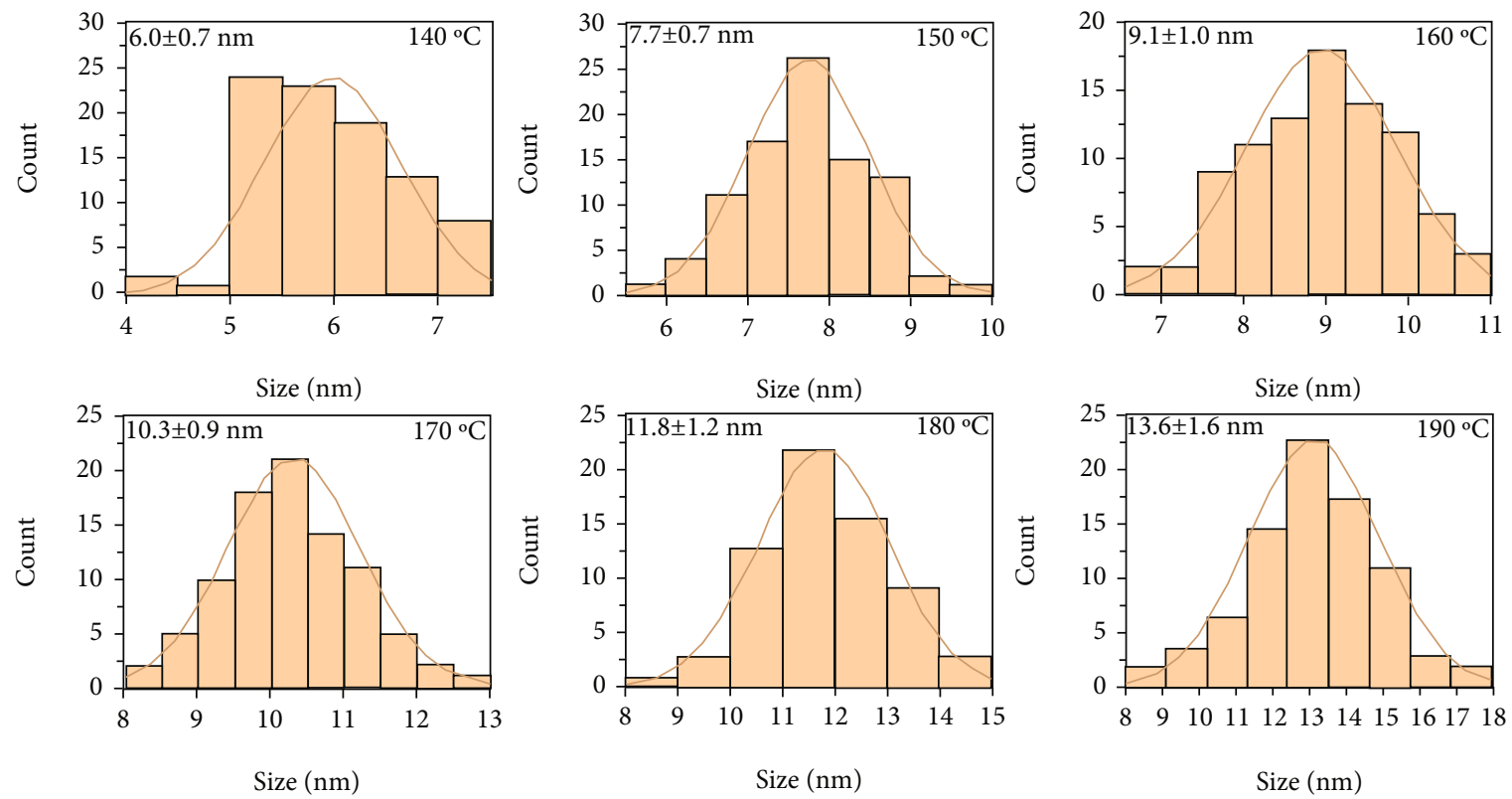

FIgure 2: The particle size distribution of PbSe CQDs at different reaction temperature.

2.2. Synthesis of PbSe Colloidal Quantum Dots. The Se-TOP solution was prepared by stirring $1.28 \mathrm{~g}$ Se in $12.8 \mathrm{~mL}$ of TOP until dissolved, and a three-neck flask is charged with $8 \mathrm{mmol}$ of $\mathrm{PbO}, 20 \mathrm{mmol}$ of $\mathrm{OA}$, and $32.56 \mathrm{~mL}$ of $\mathrm{ODE}$ and heated in a vacuum. Then, the flask is filled with $A r_{2}$, and the temperature is raised to reaction temperature; it is usually from $140^{\circ} \mathrm{C}$ to $190^{\circ} \mathrm{C} .12 .8 \mathrm{~mL}$ of the Se-TOP solution that was rapidly injected into the vigorously stirred and hot lead oleate solution. Then, PbSe CQDs are formed immediately after injecting TOP. After the reaction was terminated, the temperature of the solution was lowered by transferring the flask into an ice-water bath. The fabricated PbSe CQDs were washed two times by sequential centrifugation with anhydrous ethanol and hexane. Finally, the PbSe CQDs were dispersed in cyclohexane for use.

2.3. Device Fabrication. A cross finger electrode with a channel length of $5 \mu \mathrm{m}$ was used to fabricate photodetector, and PbSe CQDs were dissolved in cyclohexane having a concentration of $20 \mathrm{mg} \cdot \mathrm{mL}^{-1}$ and then spin-coated on the substrate to act as an active layer. For ligand exchange, a drop of 1,2ethanedithiol and hydrochloric acid were dissolved in ethanol with a concentration of $2 \%$, respectively, which was then dropped onto the PbSe film and left for 1 minute, followed by spin-coating for 20 seconds. At last, the PbSe film was washed once with methanol and ethanol. The above steps were repeated several times to obtain either EDT-PbSe or CQD thin film.

2.4. Characterization. The UV-Vis-NIR absorption spectra of PbSe CQD film were recorded using a UV/VIS/NIR Spectrophotometer (PerkinElmer, Lambda 950). The Fourier transform infrared absorption (FT-IR) spectra (Bruker, Vertex70v) of the PbSe CQD film were measured to verify that the as-synthesized ligand $(\mathrm{OA})$ on PbSe CQDs were successfully exchanged. The size of the CQDs was determined by a transmission electron microscope (TEM, Talos, L120C). Photodetector device performance was investigated with a semiconductor characterization system composed of a spectrometer (DSR-F4-XIAN, Zolix, China), a precision source meter (Keysight B2901A, USA), and a lock-in amplifier (Model SR830 DSP, USA).

\section{Results and Discussion}

Figures $1(\mathrm{a})-1(\mathrm{f})$ show the TEM images of PbSe CQDs for the reaction temperature of $140^{\circ} \mathrm{C}, 150^{\circ} \mathrm{C}, 160^{\circ} \mathrm{C}, 170^{\circ} \mathrm{C}$, $180^{\circ} \mathrm{C}$, and $190^{\circ} \mathrm{C}$, respectively, where the corresponding high-resolution TEM images are presented as the insets. The reaction time of PbSe CQDs was usually kept for 5 minutes. However, as the reaction temperature rises from $140^{\circ} \mathrm{C}$ to $190^{\circ} \mathrm{C}$, the size of PbSe CQDs increases, and the synthesized PbSe CQDs aggregate into quadrilateral or round shapes. Figures 2 and 3(a) show the particle size distribution and the size of PbSe CQDs as a function of the reaction temperature. When the reaction temperature is raised from $140^{\circ} \mathrm{C}$ to $190^{\circ} \mathrm{C}$, the crystal size increased from 6 to $15 \mathrm{~nm}$. The average particle size and standard deviation of PbSe CQDs expand as the reaction temperature increases. This indicates that in the case of lower reaction temperatures, the size dispersion of the synthesized quantum dots is smaller.

Figure 3(b) shows the X-ray diffraction (XRD) spectrum of PbSe CQDs synthesized at various reaction temperatures. The XRD peaks at $25.2^{\circ}, 29.1^{\circ}, 41.6^{\circ}, 49.3^{\circ}, 51.6^{\circ}, 60.4^{\circ}, 68.4^{\circ}$, and $76.0^{\circ}$ correspond to the (111), (200), (220), (311), (222), (400), (420), and (422) crystal planes, respectively. It can be seen that the full width at half maxima (FWHM) of diffraction peak decreases with the increase of temperature. According to Scherrer's formula, the crystal size is inversely 


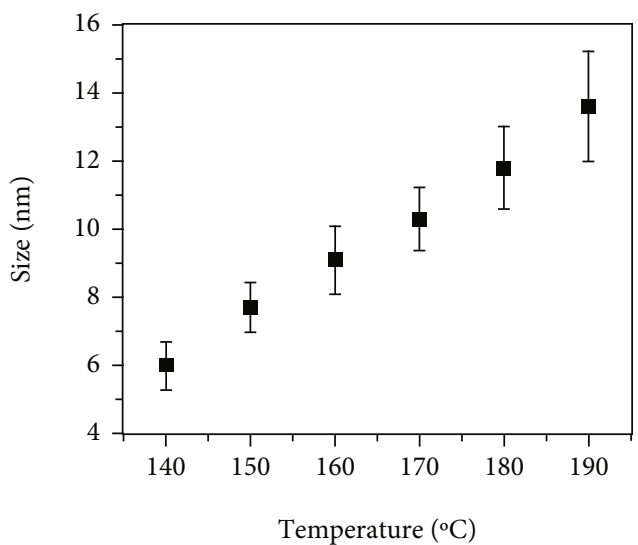

(a)

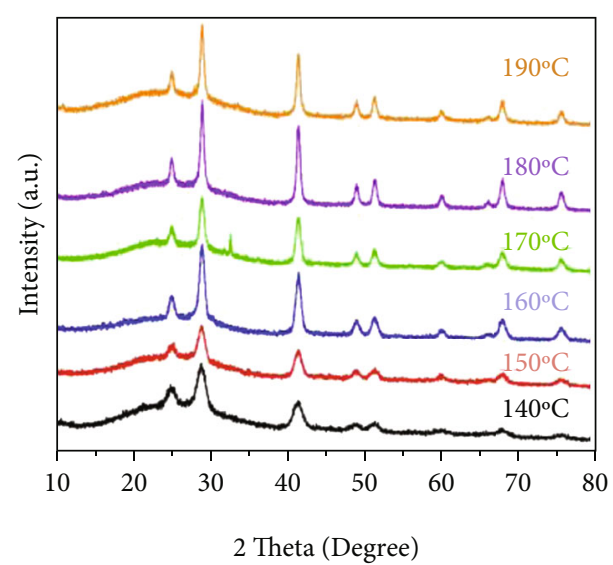

(b)

FIgure 3: (a) Plot of PbSe CQD size with respect to reaction temperature. The error bars represent the standard deviation of measurements for 90 particles. (b) XRD pattern of the PbSe CQD power.
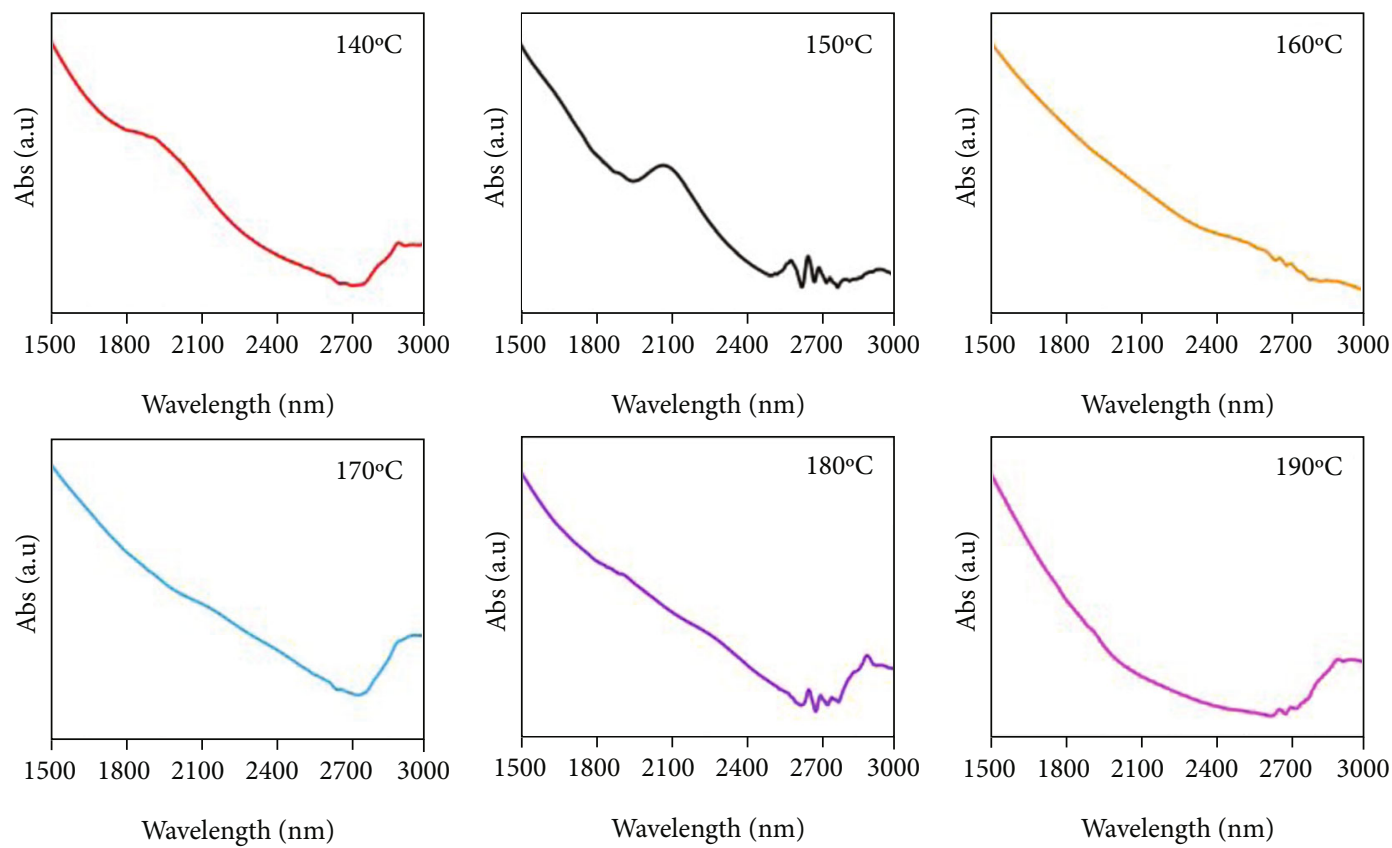

Figure 4: Optical absorption spectrum of PbSe CQDs synthesized with different reaction temperature.

proportional to the FWHM of the diffraction peak. Therefore, the TEM images and XRD spectrum confirmed that the synthesized compounds are PbSe CQDs and that the size of the PbSe CQD size increases with reaction temperature.

According to the quantum limiting effect, the first exciton absorption peak shows a red shift as the size of the quantum dot increases. Figure 4 shows the absorbance spectra of the PbSe CQD films deposited using ligand exchange process onto the $\mathrm{BaF}_{2}$ glass at the different reaction temperatures. No obvious absorption peak can be seen, which is due to the flattening of the first exciton absorption peak after the quantum dots are prepared into films and ligand exchange is performed. The first exciton absorption peak is shifted from $1900 \mathrm{~nm}$ to $2500 \mathrm{~nm}$. When the reaction tem- perature is increased, the first exciton absorption peak is even after $3000 \mathrm{~nm}$.

When fabricating PbSe colloidal quantum dots (CQDs), we always use ligands with long alkyl chains [16]. Although the presence of long-chain ligands protects the CQDs from oxidation and allows them to disperse well in solution after synthesis, it inhibits CQD-to-CQD carrier transport. Therefore, we replace the OA long-chain ligands with the EDT short-chain ligands to improve the conductivity of CQD film. The ligand exchange process is achieved using the spincoating method. As shown in Figure 5(a), the intensity of $\mathrm{C}-\mathrm{H}$ stretching absorption is reduced after the ligand exchange, indicating that the carbon in the film has been largely removed. 

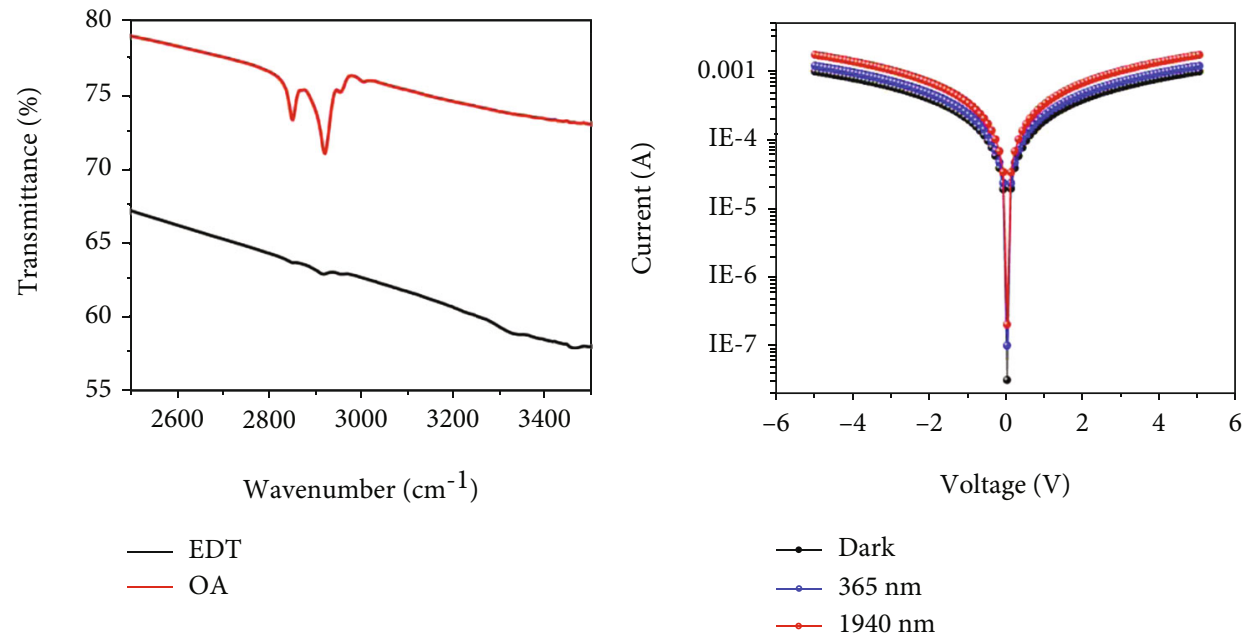

(a)

(b)

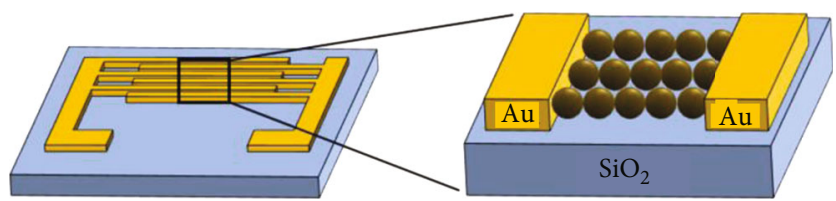

(c)

FIgure 5: (a) FTIR spectra of the PbSe CQD films with OA ligand and EDT ligand. (b) I-V curve of device in the dark and under light illumination (365 $\mathrm{nm}$ and $1940 \mathrm{~nm}$ ). (c) Schematic diagram of the PbSe CQD photodetector structure.

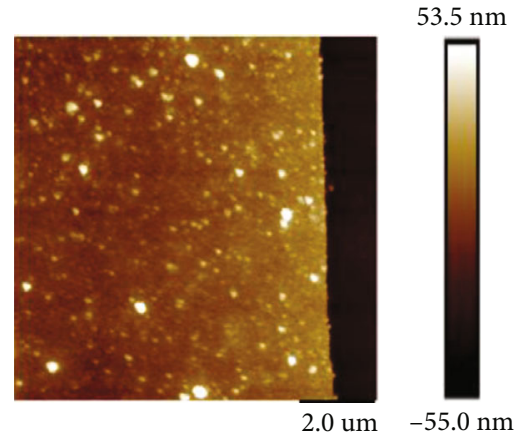

(a)

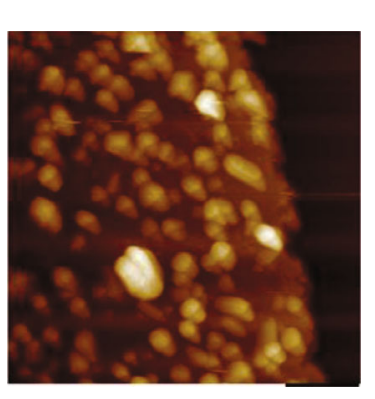

$2.0 \mathrm{um} \quad-298.9 \mathrm{~nm}$

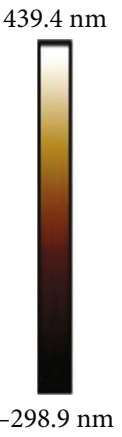

(c)

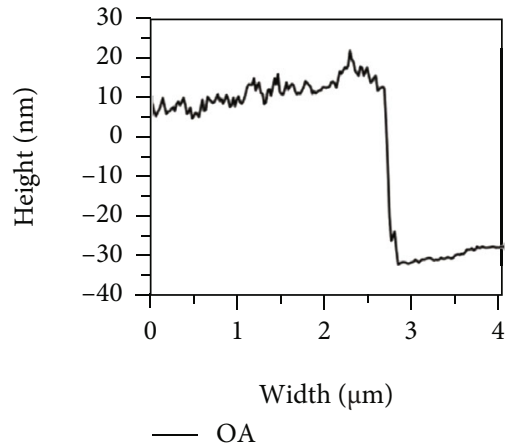

(b)

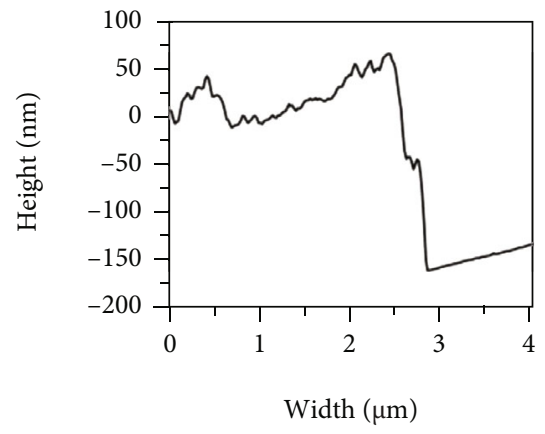

— EDT

FIgure 6: AFM 2D height profile images of PbSe film on $\mathrm{Si}_{\mathrm{SiO}}$, (a) five-layer PbSe CQD film, (b) is the curves of step height and (c) five-layer $\mathrm{PbSe}$ CQD film after exchange ligand, and (d) is the curve of step height. 


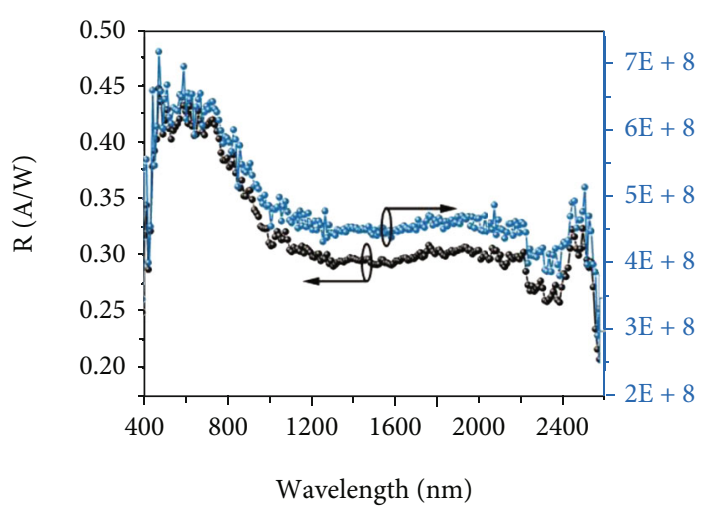

(a)

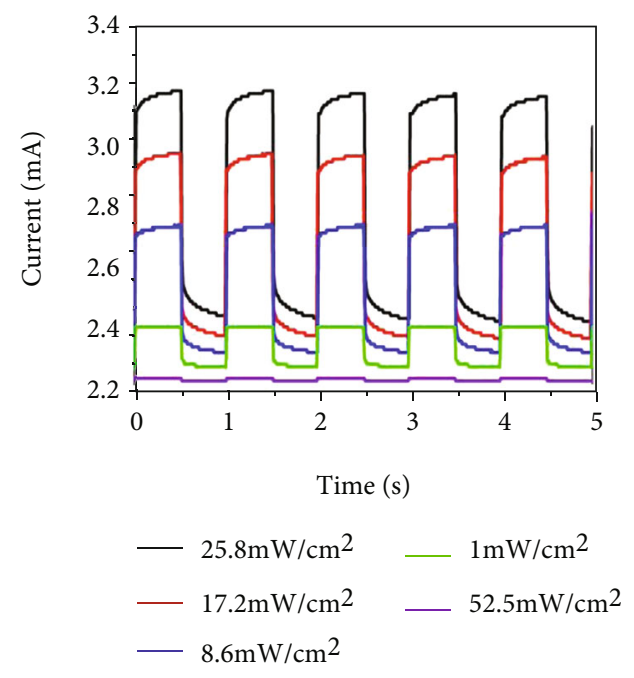

(c)

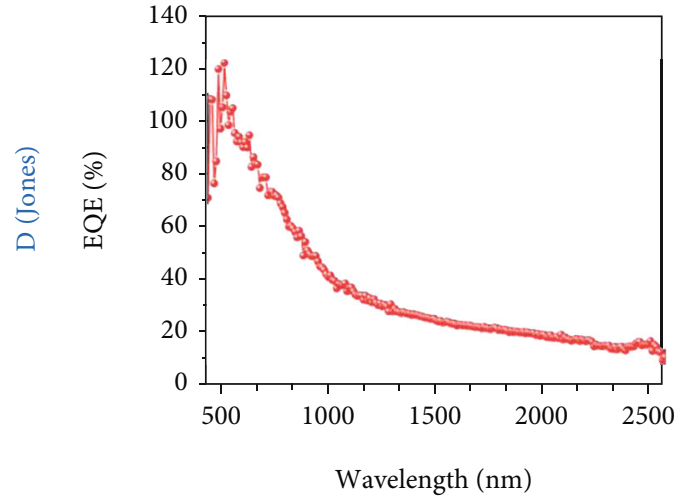

(b)

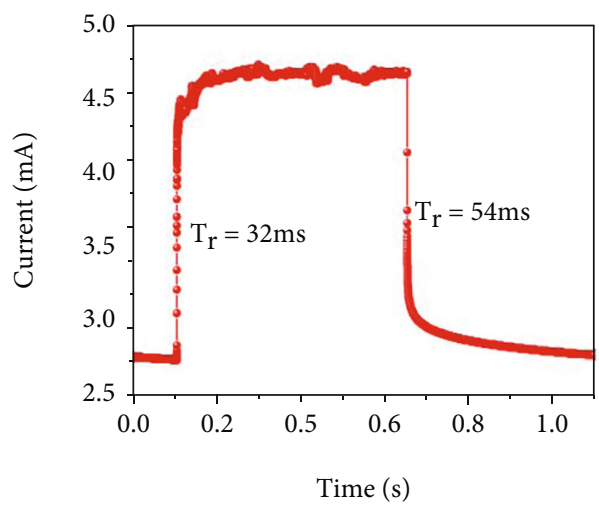

(d)

Figure 7: (a) The photoresponsivity $(R)$ and detectivity $\left(D^{*}\right)$ of the photodetector with the relationship of wavelength ranging from $400 \mathrm{~nm}$ to $2600 \mathrm{~nm}$ at $V=5 \mathrm{~V}$. (b) EQE of the PbSe CQD photodetector. (c) Photoresponse of the PbSe CQD photodetector under varied light intensity. (d) Rising and falling edges for estimating rise time $\left(T_{\mathrm{r}}\right)$ and the fall time $\left(T_{\mathrm{f}}\right)$.

It is well known that the thicker PbSe CQD film absorbs more photons, which in turn produce more carriers. We can increase the thickness of CQD film just by increasing the number of spin coats. The thickness of the PbSe CQD film was characterized by Atomic Force Microscopy (AFM). The height profiles between the PbSe CQD film and the silicon dioxide wafer are shown in Figures 6(a)and 6(b), which are step height curves. As it can be seen from Figure 6(b), the thickness of the five layers of the PbSe CQD film without ligand exchange is $\sim 50 \mathrm{~nm}$. Figures 6 (c) and $6(\mathrm{~d})$ show the step height curves of the PbSe CQD film after the ligand exchange process. As it can be seen from Figure $6(\mathrm{~d})$, the thickness of the five layers of the PbSe CQD film is $\sim 180 \mathrm{~nm}$ after the ligand exchange process. The thickness of PbSe CQD film is significantly different before and after ligand exchange during the same procedure. Thus, ligand exchange not only improves the conductivity of the film but also increases its thickness.

For photodetectors, a broadband spectrum absorption range of the active layer materials is highly desirable. The responsivity $(R)$ is another important factor that can describe the light-current transformation efficiency of the photodetector [17]. It can be determined by the amount of photo-generated current $I_{\text {ill }}$ per incident light power $P$ as

$$
R=\frac{I_{\text {light }}-I_{\text {dark }}}{P}=\frac{I_{\text {ill }}}{P},
$$

where $I_{\text {light }}$ and $I_{\text {dark }}$ refer to the current flowing through the detector under illumination and in the darkness, respectively. Furthermore, the detectivity $\left(D^{*}\right)$ is used to evaluate the efficiency of the photodetector, which is given as

$$
D^{*}=\frac{R \sqrt{A}}{\sqrt{2 q I_{\mathrm{dark}}}},
$$

where $A$ is the effective area under illumination, $q$ is the electron charge, and $I_{\text {dark }}$ is the current in darkness. Higher $D^{*}$ can be achieved at the weaker incident illumination, and the strength of the illumination is inversely proportional to $D^{*}$. It is obvious from Equation (2) that the photodetector configuration can be optimized to achieve the greater value of $D^{*}$. 
TABLE 1: The comparison of the performance of serval photodetectors now.

\begin{tabular}{lcccccc}
\hline Material & Type & Spectral rang $(\mu \mathrm{m})$ & Responsibility $(\mathrm{A} / \mathrm{W})$ & Response time $(\mathrm{ms})$ & Voltage $(\mathrm{V})$ & Ref. \\
\hline PbSe CQDs & Photoconductor & $0.4-2.6$ & $0.32($ at $2520 \mathrm{~nm})$ & 32 & 5 & This work \\
Graphene-Si & Photodiode & $0.38-1.1$ & $0.45($ at $780 \mathrm{~nm})$ & 0.06 & 3 & {$[5]$} \\
Graphene-Si & Phototransistor & $0.2-1.1$ & $0.4($ at $885 \mathrm{~nm})$ & & -2 & {$[18]$} \\
Si:CQD & Photodiode & $0.4-1.6$ & $0.22($ at $1490 \mathrm{~nm})$ & 0.047 & -0.25 & {$[14]$} \\
InGaAs & Phototransistor & $0.92-1.7$ & $0.52($ at $1550 \mathrm{~nm})$ & 0.266 & 4 & {$[19]$} \\
HgCdTe & Photodiode & $2-10$ & $3($ at $6.75 \mu \mathrm{m})$ & & -0.4 & {$[20]$} \\
\hline
\end{tabular}

A photoconductive device is fabricated using the synthesized PbSe CQDs with the first exciton absorption peak of $2500 \mathrm{~nm}$ to demonstrate that they are sensitive to the photons having a wavelength in the range of $400-2500 \mathrm{~nm}$. The structure of the photoconductive device and the measurements of some of its key parameters are shown in Figure 5(c). The device area is $6 \times 4 \mathrm{~mm}^{2}$, and effective photosensitive area is $0.39 \mathrm{~mm}^{2}$. In order to further investigate the electrical characteristics of the device, current-voltage $(\mathrm{I}-\mathrm{V})$ curves of the device were obtained in the dark and under $365 \mathrm{~nm}\left(36 \mathrm{~mW} / \mathrm{cm}^{2}\right)$ and $1940 \mathrm{~nm}\left(42 \mathrm{~mW} / \mathrm{cm}^{2}\right)$ light irradiation and are shown in Figure 5(b). The current on/off ratio of 1.2 obtained by the device under $365 \mathrm{~nm}$ at a bias voltage of $5 \mathrm{~V}$, the device performed with off ratio is 1.7 under $1940 \mathrm{~nm}$ at a bias voltage of $5 \mathrm{~V}$. The PbSe CQD absorption of photons by this traditional structure produces detectable electrons, which demonstrates that the fabricated photodetector has a quite good response even in low-light conditions. The dependence of responsivity $(R)$ and detectivity $\left(D^{*}\right)$ on the photon wavelength is shown in Figure 7(a). The photodetector exhibits a broadband photoresponse $(400-2600 \mathrm{~nm})$ that shows that it is suitable for visible and near-infrared light detection. The device has a maximum responsivity of $450 \mathrm{~mA} / \mathrm{W}$ at $480 \mathrm{~nm}$, and its responsivity is about $320 \mathrm{~mA} / \mathrm{W}$ even at $2520 \mathrm{~nm}$. Its detectivity was found to be high at $2520 \mathrm{~nm}$, with a value of approximately $4.8 \times 10^{8}$ Jones.

The measured EQE of PbSe CQD photodetector is shown in Figure 7(b), which also shows a wide-band optical response in the range of $400-2600 \mathrm{~nm}$. Its highest EQE is $122 \%$ at $480 \mathrm{~nm}$ and has over $14 \%$ external quantum efficiency in the near-infrared region. The illumination intensity is a key factor in influencing the photocurrent in the photodetector. The illumination intensity-dependent light response of PbSe CQDs was further investigated, and the results are shown in Figure 7(c). When the power intensity of the optical signal is increased from $52.5 \mu \mathrm{W} / \mathrm{cm}^{2}$ to $25.8 \mathrm{~mW} / \mathrm{cm}^{2}$, the optical response is clearly improved, and the optical signal has good sensitivity.

The response time is usually defined by the rise and the fall times, which are the times between $10 \%$ and $90 \%$ of the rising edge and $90 \%$ and $10 \%$ of the falling edge of the optical pulse response curve, respectively. When light is illuminated on the surface of the photodetector, the generated carriers are injected into the electrode, and the photodetector responds quickly. However, with the increase of carriers, the response of the photodetector gradually slows down until it reaches stability. As shown in Figure 7(d), the rise time of the photodetector is $32 \mathrm{~ms}$ but as the incident light is turned off, its response drops rapidly, resulting in a fall time of $54 \mathrm{~ms}$. Then, we compare various performance parameters of $\mathrm{PbSe} C Q D$ photodetector with several kinds of the traditional photodetector in Table 1. As can be seen from the data in the table, $\mathrm{PbSe}$ CQD photodetector has a wide spectral range among several detectors, and it also has a responsivity of $0.32 \mathrm{~A} / \mathrm{W}$ at $2520 \mathrm{~nm}$.

\section{Conclusions}

Lead selenide quantum dots have been successfully synthesized on a large area with the help of a low-cost, solution process. The reaction temperature of $\mathrm{PbSe}$ colloidal quantum dots has been used to tune the size of colloidal quantum dots, and the relationship between the size of quantum dots and the reaction temperature was obtained. The oleic acid ligand was successfully replaced with 1,2-ethanedithiol. The films of $\mathrm{PbSe}$ CQDs were deposited using a layer-by-layer (LbL) spincoating method and utilized to fabricate a photoconductive device. The device showed a broad spectrum response ranging from $400 \mathrm{~nm}$ to $2600 \mathrm{~nm}$. The photodetector has a quite good response of $320 \mathrm{~mA} / \mathrm{W}$ even at $2520 \mathrm{~nm}$ in room temperature conditions. The photodetector has more than $14 \%$ external quantum efficiency (EQE) in the short-wavelength infrared region. The excellent performance exhibited by the photodetector fabricated by PbSe CQDs indicates that this material has a promising future in optoelectronic industry.

\section{Data Availability}

No data were used to support this study.

\section{Conflicts of Interest}

The authors declare no conflict of interest.

\section{Acknowledgments}

This research was funded by the Key Research and Development Program of Shaanxi Province (2019ZDLGY16-1).

\section{References}

[1] C. Dong, S. Liu, N. Barange et al., "Long-wavelength lead sulfide quantum dots sensing up to $2600 \mathrm{~nm}$ for short-wavelength infrared photodetectors," ACS Applied Materials \& Interfaces, vol. 11, no. 47, pp. 44451-44457, 2019. 
[2] X. Hu, X. Zhang, L. Liang et al., "High-performance flexible broadband photodetector based on organolead halide perovskite," Advanced Functional Materials, vol. 24, no. 46, pp. 7373-7380, 2014

[3] J. Y. Zhang, J. L. Xu, T. Chen, X. Gao, and S. D. Wang, "Toward broadband imaging: surface-engineered $\mathrm{PbS}$ quantum dot/perovskite composite integrated ultrasensitive photodetectors," ACS Applied Materials \& Interfaces, vol. 11, no. 47, pp. 44430-44437, 2019.

[4] L. Gao, C. Chen, K. Zeng et al., "Broadband, sensitive and spectrally distinctive $\mathrm{SnS}_{2}$ nanosheet/PbS colloidal quantum dot hybrid photodetector," Light: Science \& Applications, vol. 5, no. 7, article e16126, 2016.

[5] J. Zhao, H. Liu, L. Deng et al., "High quantum efficiency and broadband photodetector based on graphene/silicon nanometer truncated cone arrays," Sensors, vol. 21, no. 18, 2021.

[6] J. Hu, S. Yang, Z. Zhang et al., "Solution-processed, flexible and broadband photodetector based on $\mathrm{CsPbBr}_{3} / \mathrm{PbSe}$ quantum dot heterostructures," Journal of Materials Science \& Technology, vol. 68, pp. 216-226, 2021.

[7] S. Wen, W. Liu, H. Liu, and F. Xie, "Synthesis of HgTe quantum dots and its application in broadband photodetection," in 2021 International Conference of Optical Imaging and Measurement (ICOIM), pp. 45-48, Xi'an, China, 2021.

[8] J. Song, J. Li, X. Li, L. Xu, Y. Dong, and H. Zeng, "Quantum dot light-emitting diodes based on inorganic perovskite cesium lead halides (CsPbX3)," Advanced Materials, vol. 27, no. 44, pp. 7162-7167, 2015.

[9] Y. Wei, Z. Ren, A. Zhang et al., "Hybrid organic/PbS quantum dot bilayer photodetector with low dark current and high detectivity," Advanced Functional Materials, vol. 28, no. 11, 2018.

[10] J. Hu, Y. Shi, Z. Zhang, R. Zhi, S. Yang, and B. Zou, "Recent progress of infrared photodetectors based on lead chalcogenide colloidal quantum dots," Chinese Physics B, vol. 28, no. 2, article 020701, 2019.

[11] T. Nakotte, H. Luo, and J. Pietryga, "PbE (E = S, Se) colloidal quantum dot-layered 2D material hybrid photodetectors," Nanomaterials, vol. 10, no. 1, 2020.

[12] H. Wang, Z. Li, C. Fu et al., "Solution-processed PbSe colloidal quantum dot-based near-infrared photodetector," IEEE Photonics Technology Letters, vol. 27, no. 6, pp. 612-615, 2015.

[13] Y. Zhang, C. Ding, G. Wu et al., "Air stable PbSe colloidal quantum dot heterojunction solar cells: ligand-dependent exciton dissociation, recombination, photovoltaic property, and stability," The Journal of Physical Chemistry C, vol. 120, no. 50, pp. 28509-28518, 2016.

[14] X. Xiao, K. Xu, M. Yin et al., "High quality silicon: colloidal quantum dot heterojunction based infrared photodetector," Applied Physics Letters, vol. 116, no. 10, p. 101102, 2020.

[15] C. Fu, H. Wang, T. Song et al., "Stability enhancement of PbSe quantum dots via post-synthetic ammonium chloride treatment for a high-performance infrared photodetector," Nanotechnology, vol. 27, no. 6, article 065201, 2016.

[16] W. Hu, S. Gao, P. N. Prasad, J. Wang, and J. Xu, "Employing photoassisted ligand exchange technique in layered quantum dot LEDs," Journal of Nanomaterials, vol. 2012, 5 pages, 2012.

[17] M. Liao, "Progress in semiconductor diamond photodetectors and MEMS sensors," Functional Diamond, vol. 1, no. 1, pp. 29-46, 2021.
[18] X. An, F. Liu, Y. J. Jung, and S. Kar, "Tunable graphene-silicon heterojunctions for ultrasensitive photodetection," Nano Letters, vol. 13, no. 3, pp. 909-916, 2013.

[19] Q. Yang, Q. Wu, W. Luo, W. Yao, S. Yan, and J. Shen, "InGaAs/graphene infrared photodetectors with enhanced responsivity," Materials Research Express, vol. 6, no. 11, 2019.

[20] P. Martyniuk, W. Gawron, P. Madejczyk, A. Rogalski, and J. Piotrowski, "Modeling of HgCdTe LWIR detector for high operation temperature conditions," Metrology and Measurement Systems, vol. 20, no. 2, pp. 159-170, 2013. 